\title{
Interchain effects in the ultrafast photophysics of a semiconducting polymer: THz time-domain spectroscopy of thin films and isolated chains in solution
}

\author{
E. Hendry, ${ }^{1,2, *}$ M. Koeberg, ${ }^{1,2}$ J. M. Schins ${ }^{3}$ H. K. Nienhuys, ${ }^{4}$ V. Sundström, ${ }^{4}$ L. D. A. Siebbeles, ${ }^{3}$ and M. Bonn ${ }^{1,2}$ \\ ${ }^{1}$ Leiden Institute of Chemistry, Leiden University, P.O. Box 9502, 2300 RA Leiden, The Netherlands \\ ${ }^{2}$ FOM Institute for Atomic and Molecular Physics, Kruislaan 407, 1098 SJ, Amsterdam, The Netherlands \\ ${ }^{3}$ Interfaculty Reactor Institute, Delft University of Technology, Mekelweg 15, $2629 \mathrm{JB}$ Delft, The Netherlands \\ ${ }^{4}$ Department of Chemical Physics, Chemical Center, Lund University, Getingevägen 60, 22241 Lund, Sweden
}

(Received 20 August 2004; revised manuscript received 18 October 2004; published 2 March 2005)

\begin{abstract}
We compare the generation and decay dynamics of charges and excitons in a model polymer semiconductor (MEH-PPV) in solution and drop-cast thin films, by recording the sub-ps transient complex conductivity using $\mathrm{THz}$ time-domain spectroscopy. The results show that the quantum efficiency of charge generation is two orders of magnitude smaller in solution $\left(\sim 10^{-5}\right)$ than in the solid film $\left(\sim 10^{-3}\right)$. The proximity of neighboring chains in the films apparently facilitates (hot) exciton dissociation, presumably by allowing the electron and hole to separate on different polymer strands. For both samples, photoexcitation leads to the predominant formation of bound charge pairs (excitons) that can be detected through their polarizability. Surprisingly, the polarizability per absorbed photon is a factor of 3 larger in solution than in the film, suggesting that interchain interactions in the film do not result in a substantial delocalization of the exciton wave function.
\end{abstract}

DOI: 10.1103/PhysRevB.71.125201

PACS number(s): 72.80.Le, 73.50.Gr, 71.35.Aa, 68.55.Jk

\section{INTRODUCTION}

Conjugated semiconducting polymers are increasingly finding applications in optoelectronic devices such as lightemitting diodes (LED's) and solar cells. ${ }^{1}$ Despite their apparent technological importance, many of the fundamental aspects of photoexcitation processes in these materials, which determine many of the properties of such optoelectronic devices, have remained the subject of intense debate. One issue that has received particular attention the past few years is the effect of polymer morphology on charge and energy transfer (an excellent review is provided in Ref. 2).

The sample morphology determines to what extent the polymer chains constituting the sample can interact. Such interchain interactions can result in charge transfer between polymer strands, which is essential to charge transport across macroscopic distances, as the length of one polymer strand is limited. ${ }^{2}$ Indeed, it has been suggested that interchain charge transport limits the overall charge mobility, ${ }^{3}$ by noting that charge mobility within one single chain is much higher than within an amorphous film. ${ }^{4}$ For excitons - the primary photoexcitation product of semiconducting polymers ${ }^{5}$ - the delocalization over different chains is adverse to the use of these materials in electroluminescent devices, as interchain excitons are intrinsically weakly emissive. ${ }^{6}$

It has been proposed that up to $90 \%$ of the excitations in thin-film samples result in interchain species, ${ }^{7,8}$ with the largest part of the electron and hole wave functions residing on separate polymer chains, though this is certainly controversial. ${ }^{2,9}$ Theoretical modeling has recently shown that interchain effects are particularly important in closepacked polymer crystals. ${ }^{10}$ However, most polymer solid samples are generally amorphous and disordered, as the large polymer molecules are very difficult to crystallize. This leads to a structure with a large variance in chain-chain distances ${ }^{11}$ [see Fig. 1(a)], with only a small fraction of the polymer chain length "close" to neighboring chains. Hence, solid polymer films are largely somewhere in between closepacked crystalline samples and solutions [shown in Fig. 1(b)], where the polymer chains are, on average, far enough apart to be considered isolated.
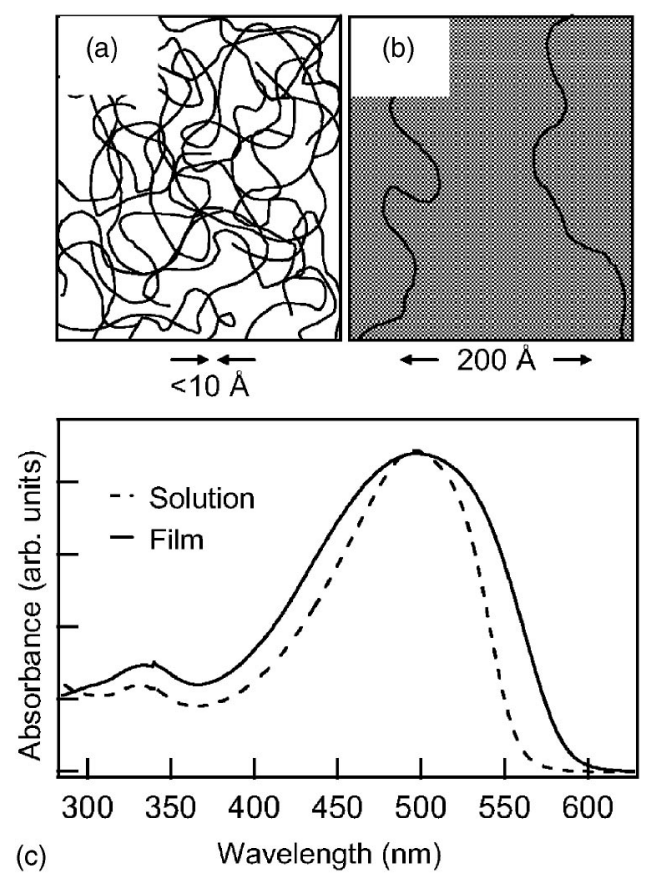

FIG. 1. Pictorial representation of sample morphology for (a) film and (b) benzene solution samples. In the film, the nearestneighbor chain distance can be as small as $4 \AA$ (Ref. 27). Comparison between the two samples allows the study of interchain effects on the photophysics of these materials. (c) Normalized UV-VIS absorption spectra for a solution and a film sample. The broader absorption of the film sample suggests a more disordered state. 
As a result of these considerations, the role of the morphology on the semiconducting polymer's optical, electrical and electro-optic properties has been the subject of many studies; see, e.g., Refs. 2 and 12-14. Many such studies make use of the change in optical transitions upon changing the polymer morphology. Indeed, the UV-VIS spectra of benzene solution and thin-film samples are very different [see Fig. 1(c)]. Here, the broadened absorption of the film sample suggests that the thin-film phase is more disordered than the solution. ${ }^{15}$ Indeed, the strong interaction with "good" solvents, such as benzene, allows the polymer to adopt an open, extended chain conformation in solution. ${ }^{16}$ The polymer fluorescence can also be redshifted as a result of interchain interactions; the exciton energy levels are lowered when the exciton becomes increasingly delocalized. Ultrafast time-domain studies have provided additional insights into morphological effects on dynamical processes after photoexcitation-e.g., by comparing the excited-state absorption dynamics to the fluorescence decay ${ }^{17}$ - and variations in photoluminescence. ${ }^{18}$ It has also been demonstrated, however, that such optical studies may be complicated by the fact that different types of excitons and charges may have very similar spectral signatures. ${ }^{18-22} \mathrm{THz}$ time-domain spectroscopy (THz-TDS) therefore ideally complements optical studies, as it allows one to directly probe both exciton and free charge species simultaneously: it is sensitive to the real and imaginary components of frequency-dependent $(0.2-1.5 \mathrm{THz})$ photoconductivity and, as such, can monitor free and bound charges on a subpicosecond timescale. Our initial THz-TDS studies ${ }^{5}$ of semiconducting polymers have indicated that excitons are the primary photoexcitation product, while only a small number of free charges are formed on ultrafast times, most likely from dissociation of "hot" excitons. ${ }^{23-25}$ In this picture, nascent excitons in excited states can use their excess energy, before cooling ( $\sim 100 \mathrm{fs}),{ }^{24,25}$ to overcome the dissociation barrier.

Here, we use THz-TDS to follow the subpicosecond excitation process in two phases of the model semiconducting polymer poly [2-methoxy-5-(2'ethyl-hexyloxy) -1,4-phenylene vinylene] (MEH-PPV). In solution the polymer concentration is sufficiently low and the solvation (in benzene) is sufficiently strong, ${ }^{26}$ so that we can study this process on essentially isolated polymer chains. In the thin-film phase interchain interactions are present. ${ }^{2,6,8,11,18,27,28}$ On short (subpicosecond) times, only a small number $(<1 \%)$ of free charges is generated in the solid film, while this number is even smaller (by approximately 2 orders of magnitude) in solution. On longer timescales ( $>5 \mathrm{ps})$ mobile charges are not observed and the photoinduced changes in the $\mathrm{THz}$ wave form are mainly due to the polarizability of excitons. Furthermore, in the film sample, the restricted motion of the polymer chains and the stronger effect of torsional disorder limit the exciton size and reduce the exciton polarizability by a factor of 3. The possible effects of interchain species $^{2,6,14,18,27}$ on the observed excitation properties are discussed.

\section{EXPERIMENTAL PROCEDURE}

MEH-PPV films of $\sim 20 \mu \mathrm{m}$ thickness are prepared by drop casting the polymer from a benzene solution onto

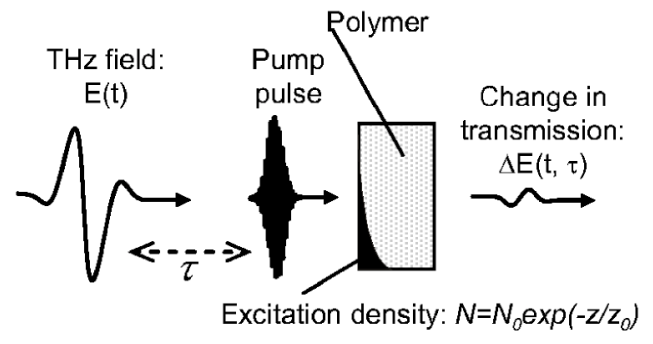

FIG. 2. The measurement involves measuring the pump-induced change in transmission, $\Delta E(t, \tau)$, as a function of delay, $\tau$, between pump and THz probe pulses, $E(t)$, and the 400 -nm pump pulse. The 400-nm excitation results in a exponentially decaying density $N(z)$ in the samples, characterized by a skin depth $z_{0} \sim 3 \mathrm{~mm}$ in solution and $\sim 100 \mathrm{~nm}$ in the film (Ref. 43 ).

water-free quartz plates, using dry polymer from Sigma Aldrich. All measurements on film samples are performed under vacuum $\left(<10^{-3}\right.$ mbar $)$ to impede photo-oxidation. Films produced in this manner have average chain-chain distances $<10 \AA$ (Ref. 27). Solution samples are made in water-free benzene of concentration $2.6 \mathrm{~g} / 1$ (corresponding to an average chain-chain separation $\sim 200 \AA$ ). Benzene was chosen as it is transparent to $\mathrm{THz}$ radiation and is a good solvent for MEH-PPV. ${ }^{26}$ Photo-oxidation of the polymer in solution is abated by using a quartz flow cell. Photoexcitation is accomplished with 400-nm (3-eV), 150-fs full width at half maximum (FWHM) laser pulses, allowing excitation above the absorption gap of MEH-PPV, which is located at $2.5 \mathrm{eV}$ (Ref. 29). Unless stated otherwise, the measurements presented here were performed with an excitation fluence of $60 \mathrm{~J} \mathrm{~m}^{-2}$.

The THz setup is similar to that described in Ref. 30. The field strength $E(t)$ transmitted through the sample is measured directly in the time domain, through electro-optical sampling with $800-\mathrm{nm}, 150-$ fs pulses: this "gating" technique allows a time resolution better than the $\mathrm{THz}$ pulse duration, ${ }^{31}$ permitting the study of the transient photoconductivity in these materials with subpicosecond temporal resolution and allowing us to probe the excitation process in polymers in real time. ${ }^{5}$ In contrast to conventional transientabsorption studies, in which generally only the amplitude of the probe beam is recorded, in $\mathrm{THz}$ studies both the amplitude and the phase of the THz field $E(t)$ are determined. This allows for the extraction of both the real and imaginary parts of the complex conductivity over a wide frequency range. Following optical excitation, we measure the pump-induced modulation $\Delta E(t, \tau)$ of the transmitted $\mathrm{THz}$ field as a function of delay $\tau$ between pump and $\mathrm{THz}$ probe pulses-see Fig. 2. The THz pulses are essentially single-cycle electromagnetic pulses of about 1 ps FWHM, as depicted in the upper panel of Fig. 3. The lower panel in Fig. 3 shows $\Delta E(t, \tau)$ (Refs. 30 and 31) - the modulation of the transmitted $\mathrm{THz}$ field (measured here for the solution sample) as a function of detection pulse delay $t$ and excitation pulse delay $\tau$. In Fig. 3, $\tau=0$ is chosen to be the pump pulse delay where a modulated signal is first observed. At all delays the modulation is $<1 \%$ of the weak incident THz electric field (peak field $>1 \mathrm{kV} / \mathrm{cm}$ ). It should be noted that these fields are 


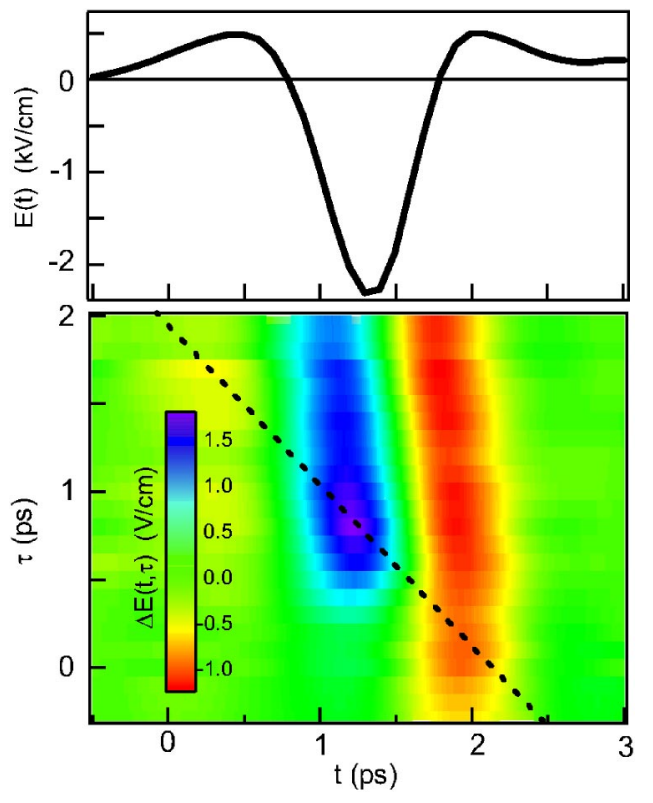

FIG. 3. (Color online) A measurement taken on the solution sample: $E(t)$ (top) is the incident $\mathrm{THz}$ field, with FWHM $\sim 1 \mathrm{ps}$. The bottom plot presents the excitation-induced modulation $\Delta E(t, \tau)$ of the THz probe field at delay $\tau$. There is both a decrease in magnitude of $\Delta E(t, \tau)$ as function of $\tau$, indicating a decrease in conductivity with pump-probe delay. There is also a shift of the wave form to the left, becoming more like the derivative of $E(t)$ in the upper panel. This occurs as the imaginary component of the conductivity becomes more dominant [see Fig. 6(a)], as the polarizability of the excited state slows the $\mathrm{THz}$ pulse. The dotted line at a $45^{\circ}$ angle represents the path of the pump pulse in the measurement; i.e., each point in a horizontal cross section $E(t)$ has a different pump-probe delay. Since the decay of the signal is of the order of the FWHM of the THz probe pulse, this must be taken into account in the analysis (see Sec. III). Transforming the data along this line (as described in the analysis section) introduces an alternative time $\tau^{\prime}$ to describe the time between excitation pulse and all points on the probe $\mathrm{THz}$ pulse with same pump delay.

much lower than the MV/cm fields typically used in devices, so that our $\mathrm{THz}$ pulse acts as a true probe pulse, not perturbing the system. A linear dependence of signal on pump fluence is observed.

\section{ANALYSIS}

THz-TDS experiments are in some respects similar to pump-probe experiments with visible light. A visible-light pump pulse creates an excitation in a sample, which is probed with a broadband $\mathrm{THz}$ pulse at a time $\tau$ after the excitation (see Fig. 2). The strength of THz-TDS lies in the distinctive detection technique ${ }^{30,32}$ : not only the change in absorbance is determined, but also the phase shift of all frequency components contained in the probe field. This information is best expressed in terms of the complex photoconductivity spectrum $\sigma(\omega, \tau)=\sigma^{\prime}(\omega, \tau)+i \sigma^{\prime \prime}(\omega, \tau)$, which depends parametrically on time $\tau$ after photoexcitation. In principle, the experimentally determined absorption and

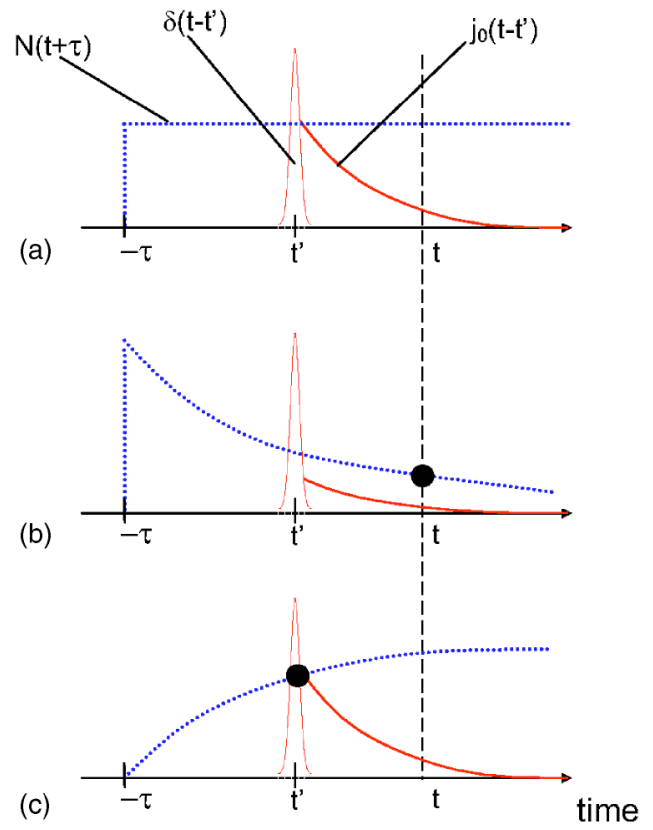

FIG. 4. (Color online) Evaluation of the current (red, solid line) from the impulse by the $\mathrm{THz}$ field [here represented, for simplicity, as a delta function field $\left.\delta\left(t-t^{\prime}\right)\right]$. The current density $J\left(\tau, t, t^{\prime}\right)$ at some time $t$ (highlighted by the vertical black, long dashed line) after excitation is separated into an amplitude function $N(t+\tau)$ (blue, dotted line) which describes the amplitude dependence on the pump delay $(t+\tau)$, and the average singleparticle response to the impulse field, $j_{0}\left(t-t^{\prime}\right)$. (a) In the case where the conductivity is independent of pump-probe delay, $N(t+\tau)$ is constant in time and the current at time $t$ is given by $J\left(\tau, t, t^{\prime}\right)=N j_{0}\left(t-t^{\prime}\right)$. (b) When the conductivity is decreasing with pump-probe delay, only the contribution of remaining particles at time $t$ (highlighted by the black dot) is to be considered, such that the current density is given by $J\left(\tau, t, t^{\prime}\right)=N(t+\tau) j_{0}\left(t-t^{\prime}\right)$. (c) When the conductivity is increasing with pump-probe delay, $J\left(\tau, t, t^{\prime}\right)$ depends on the amplitude at time $t^{\prime}$ (at the time of impulse, again highlighted by the black dot), since any additional change in amplitude after this point is unaffected by the impulse field $\delta\left(t-t^{\prime}\right)$. Then, the current density at time $t$ is given by $J\left(\tau, t, t^{\prime}\right)=N\left(t^{\prime}+\tau\right) j_{0}\left(t-t^{\prime}\right)$.

phase shift of the THz field $\Delta E(t, \tau)$ are fully and uniquely determined by $\sigma(\omega, \tau)$ (Ref. 33). However, if $\sigma(\omega, \tau)$ varies on a time scale $\tau$ that is shorter than or comparable with the THz pulse duration, the extraction of $\sigma(\omega, \tau)$ from $\Delta E(t, \tau)$ is considerably complicated, ${ }^{31,33}$ as we probe different sample properties with the beginning of the $\mathrm{THz}$ pulse than with the tail. This effect must clearly be taken into account in the analysis of data such as that presented in Fig. 3.

\section{A. Steady-state approximation}

In introducing the analysis, it is instructive to first consider the case of constant, time-independent carrier density in the film, such as the situation depicted in Fig. 4(a). The extraction of $\sigma(\omega, \tau)$ from the experimental data is straightforward if $\sigma(\omega, \tau)$ changes slowly with time $\tau$, during the $\mathrm{THz}$ probe pulse (FWHM $\sim 1 \mathrm{ps})$, since this allows for the defi- 
nition of a quasi-steady-state conductivity $\sigma(\omega)$ (Refs. 30, 34 , and 35). Before taking into account the $\mathrm{THz}$ field used in the experiment, we first consider the impulse-response current $j(t)$ after an infinitely short pulsed field $E(t)=\delta\left(t-t^{\prime}\right)$ (Ref. 36 ). We can write the impulse-response current as the product

$$
j(t)=N j_{0}(t),
$$

where $N$ is the concentration of (photoexcited) charge carriers and $j_{0}(t)$ is the single-particle current-response function. ${ }^{36}$ The sample conductivity spectrum $\sigma(\omega)$-the quantity of interest-is simply the Fourier transform of $j(t)$. When $N$ is time independent, as in this steady-state approximation, $j(t)$ is simply proportional to $j_{0}(t)$, which decays with time after the impulse by $\delta\left(t-t^{\prime}\right)$ - see Fig. 4(a). The total current $J(t)$ generated by an arbitrary $\mathrm{THz}$ pulse $E_{0}(t)$ is then obtained through the integration

$$
J(t)=N \int_{-\infty}^{\infty} E_{0}\left(t^{\prime}\right) j_{0}\left(t-t^{\prime}\right) d t^{\prime} .
$$

This is more simply written as a convolution between the time-dependent $\mathrm{THz}$ field and the impulse-response current:

$$
J(t)=N E_{0}(t) * j_{0}(t) .
$$

In the experiment, we measure the change $\Delta E(t)$ due to photoexcitation in the transmitted electrical field of the $\mathrm{THz}$ pulse. For a sample with an excitation thickness $z_{\text {slab }}$, surrounded by a medium with index of refraction $n_{T H z}$, it can be derived from the Maxwell equations ${ }^{37}$ that $\Delta E(t)$ and $J(t)$ are related through

$$
\Delta E(t)=-\frac{z_{\text {slab }}}{2 \varepsilon_{0} c n_{T H z}} J(t) .
$$

It should be noted that this solution applies only to samples with no dispersion and signals where $\Delta E(t) \ll E(t)$. The applicability of these assumptions in the present case is discussed in Sec. III D.

The sample conductivity spectrum $\sigma(\omega)$ in this steadystate approximation can be obtained straightforwardly by considering the Fourier transforms of Eqs. (2b) and (3) so that

$$
\sigma(\omega)=-\frac{2 \varepsilon_{0} c n_{T H z}}{z_{\text {slab }}} \frac{\Delta E(\omega)}{E(\omega)},
$$

where $\Delta E(\omega)$ and $E(\omega)$ are the Fourier transforms of the experimental data $\Delta E(t)$ and $E(t)$. It should be noted that the above approach is valid only if the charge-carrier concentration $N$ does not change significantly over the duration of the THz pulse ( $\sim 1$ ps), as in Refs. 30, 34, and 35 .

\section{B. Nonsteady-state conditions}

The steady-state approach in the previous section is not valid if the properties of the sample change rapidly-for example, during the excitation by the pump pulse, when the charge carriers exhibit fast trapping or recombination dynamics or when the response of charges is time dependent as a result of, for instance, carrier cooling or trapping., ${ }^{5,31,38}$ The steady-state approach indeed breaks down for the polymer samples, since the signal decay in Fig. 3 is comparable to the length of the THz pulse. Here, one would like to extract a time-dependent conductivity spectrum $\sigma(\omega, \tau)$ (Ref. 33 ) but the extraction of this quantity that is varying both with frequency and pump-probe time is not trivial, as we will show in this section.

A rapidly decaying signal, such as in Refs. 5, 31, and 39 and that observed here for the polymer samples (see Fig. 3) can be described by a time-varying charge density. In the simplified case of an infinitely short probe field $\delta\left(t-t^{\prime}\right)$, the formalism can be easily rewritten for a population $N(t+\tau)$ decaying monotonously and simultaneously in the positive direction for both $t$ and $\tau$ : In this case, we have to consider the faster decay of current in the temporal region $t^{\prime}$ to $t$ - see Fig. 4(b) - as changes after $t^{\prime}$ (the arrival time of the impulse field) do affect the experimental signal, since carriers that decay can no longer contribute to the current at time $t$. The contribution of $N(t+\tau)$ to the current at time $t$ is indicated by a black dot in Fig. 4(b). Expanding this for a density decaying monotonously across an arbitrary $\mathrm{THz}$ pulse shape, we can rewrite Eq. (2b) for decreasing density:

$$
J(t, \tau)=N(t+\tau)\left[E_{0}(t) * j_{0}(t)\right] .
$$

In order to extract the time-dependent conductivity from the data, we use a method that was introduced by Schmuttenmaer and co-workers. ${ }^{31,33}$ Prior to carrying out Fourier transforms to the time-domain data, we apply the transformation $\tau^{\prime}=t+\tau$ to Eq. (5) and obtain

$$
J_{\text {proj }}\left(t, \tau^{\prime}\right)=N\left(\tau^{\prime}\right)\left[E_{0}(t) * j_{0}(t)\right] .
$$

The experimental data are transformed along the path of the excitation pulse (shown by a dotted line in Fig. 3) so that all points on a horizontal cross section of $\Delta E_{T H z}$ now have the same pump-probe delay. The equivalent of Eq. (3) for the time-dependent density is then given by

$$
\Delta E_{p r o j}\left(t, \tau^{\prime}\right)=-\frac{z_{s l a b}}{2 \varepsilon_{0} c n} J_{p r o j}\left(t, \tau^{\prime}\right) .
$$

The conductivity spectrum $\sigma\left(\omega, \tau^{\prime}\right)$ can then be calculated, as for the steady-state case, by considering the Fourier transforms of Eqs. (6) and (7) so that

$$
\sigma_{p r o j}\left(\omega, \tau^{\prime}\right)=-\frac{2 \varepsilon_{0} c n_{T H z}}{z_{\text {slab }}} \frac{\Delta E\left(\omega, \tau^{\prime}\right)}{E(\omega)} .
$$

The dependence on the pump-probe delay $\tau^{\prime}$ is straightforward and represents the decay of the charge-carrier population. The frequency-dependent part corresponds to the response of an infinitely long-lived charge carrier.

It should be noted, however, that Eqs. (5)-(8) do not adequately describe the case where new charge carriers are being created during or after the $\mathrm{THz}$ pulse. This problem is most easily illustrated again for a delta function probe field $\delta\left(t-t^{\prime}\right)$ - see Fig. 4(c). In the case that the density is increasing between $t^{\prime}$ and $t$, the additional charges are not affected by the field at time $t^{\prime}$ and hence do not contribute to any current at time $t$. The relevant density for calculating the 
current is then $N\left(t^{\prime}+\tau\right)$ in place of $N(t+\tau)$. However, the introduction of $t^{\prime}$ dependence in $N$ means that the equivalent form of Eq. (5) for increasing density is not a simple convolution and interpretation of the extracted conductivity spectrum is not straightforward. This means that the transformation applied to the data in this paper does not give meaningful results when the pump and $\mathrm{THz}$ pulses overlap (contrary to previous claims ${ }^{31,39}$ ). For this reason, we do not consider our data for pump-THz delays $<0.5$ ps. A more detailed mathematical extraction procedure that includes the extraction of the conductivity spectrum at short times will be presented in a separate paper. ${ }^{37,40}$

\section{Detector response function}

Before the transformed data can be related to the conductivity, one further step is required: due to a limited detection bandwidth, the detected $\mathrm{THz}$ electric field is slightly distorted from that incident on the detector. This effect is accounted for using the detector response function ${ }^{32}$

$$
\begin{aligned}
f(\omega)= & {\left[\int_{-\infty}^{+\infty} E_{o p t}^{*}\left(\omega^{\prime}\right) E_{o p t}\left(\omega^{\prime}-\omega\right) d \omega^{\prime}\right] \chi_{\text {dif }}^{(2)} } \\
& \times(\omega) \frac{\exp \left(\frac{i \omega l}{c} \Delta n(\omega)\right)-1}{\frac{i \omega l}{c} \Delta n(\omega)}
\end{aligned}
$$

The first term in the product of Eq. (9) is the frequencydomain autocorrelation of the 800-nm detection pulse, described by a Gaussian FWHM 5.0 THz. The second term is the second-order susceptibility for difference frequency generation at $800 \mathrm{~nm}$ (Ref. 32). The final, and dominant, term describes the matching of the group velocity of the 800-nm detection pulse with the $\mathrm{THz}$ phase velocity at frequency $\omega$, required for efficient detection in the $\mathrm{ZnTe}$ electro-optic sen-

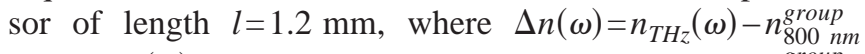

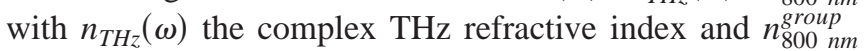
the refractive index corresponding to the group velocity at $800 \mathrm{~nm}$. We use the measured values of Gallot et al. ${ }^{41}$ for $n_{T H z}(\omega)$, while the group index at $800 \mathrm{~nm}$ in $\mathrm{ZnTe}$ has been evaluated by Bakker et al. ${ }^{42}$ to be 3.24 . The THz pulse incident on the detector is then related to the detected wave form through $E_{\text {incident }}(\omega)=E_{\operatorname{det}}(\omega) / f(\omega)$ and $\Delta E_{\text {incident }}(\omega, \tau)$ $=\Delta E_{\mathrm{det}}(\omega, \tau) / f(\omega)$. After transformation, the conductivity at different times $\tau^{\prime}$ is simply proportional to the ratio $\Delta E_{\text {incident }}\left(\omega, \tau^{\prime}\right) / E_{\text {incident }}(\omega)$ (Refs. 5, 30, and 31).

\section{General notes}

It should be noted that there are several assumptions made in the analysis described above. First, it assumes no dispersion between the propagation of visible pump and $\mathrm{THz}$ probe pulses in the excited region of the sample. This is a reasonable assumption in these experiments, since the nonpolar materials used (MEH-PPV and benzene) have very similar lowfrequency (1.78 and 1.51, respectively) and visible (1.6 and 1.5) refractive indices ${ }^{29}$, from which this time smearing ef- fect can be estimated to be at most a few femtoseconds, much shorter than the other timescales involved in the measurement. For this reason, care is also taken to ensure that the photoexcited region in the polymer is also nondispersive: this is demonstrated by the fluence independence of the extracted frequency-dependent conductivity. This requires that the modulation of the $\mathrm{THz}$ field be much smaller than the incident $\mathrm{THz}$ field itself.

Second, the solutions to Maxwell's equations presented in Eqs. (3) and (7) are exact only for a thin, homogeneously excited region and do not properly take into account effects due to multiple reflections ${ }^{35}$ in the exponentially decaying excitation region. We take these effects into account in a numerical analysis of $\mathrm{THz}$ propagation through this spatially decaying excitation region. In this calculation, the spatial decay of the signal (see Fig. 2) is described by an exponential $\sim 3 \mathrm{~mm}$ and $100 \mathrm{~nm}$ (Ref. 43) for solution and film samples, respectively, and the excitation region is discretized by splitting it into thin homogeneous slabs to approximate the smooth spatial decay of the excited state dielectric function. The $\mathrm{THz}$ pulse is subsequently propagated using the relative admittance from one slab to the next. In the solution sample these effects are negligible, since the large penetration depth of the excitation pulse into the sample $(\sim 3 \mathrm{~mm})$ leads to a very small perturbation of the refractive index of the solution at the interface. Further, any multiple reflections within the sample can simply be temporally filtered out in these measurements, as the time associated with the $\mathrm{THz}$ pulse traveling through the sample is $\sim 30 \mathrm{ps}$. In the thinfilm sample this is not the case, since the penetration depth is much smaller $[\sim 100 \mathrm{~nm}$, (Ref. 43)], though multiple reflections still contribute to less than $10 \%$ of the signal. In our analysis we take these effects fully into account.

A consequence of this extensive analysis, which takes into account the spatially varying excitation density, is that the extracted conductivities $\sigma(z)$ are also a varying function of spatial coordinate $z$. For a meaningful comparison between the different samples, we therefore discuss the conductivity at the interface $\sigma(z=0)$ normalized to the density of absorbed photons at the sample interface, defined as $\sigma_{0}=\sigma(z=0) / N_{0}$. This quantity allows for a convenient and meaningful comparison between the conductivities in the two samples with very different extinction coefficients [characterized by penetration depths of $z_{0} \sim 3 \mathrm{~mm}$ and $100 \mathrm{~nm}$ (Ref. 43) for solution and film samples, respectively]. $N_{0}$ is estimated from $z_{0}$ and the absorbed fluence $\left(40 \mathrm{~J} \mathrm{~m}^{-2}\right)$ to be $2.7 \times 10^{22} \mathrm{~m}^{-3}$ for the solution and $8 \times 10^{26} \mathrm{~m}^{-3}$ for the film. It should be noted, however, that the effects of the spatially varying excitation density are relatively small $(<10 \%)$, so that a direct evaluation of the signals through Eq. (8) for the two samples under investigation here does, in fact, provide a reasonable comparison of the spatially averaged sample conductivities.

\section{RESULTS AND DISCUSSION}

\section{A. Short-time conductivity: Free charges}

The results for subpicosecond (at $\tau^{\prime}=0.5 \mathrm{ps}$ ) conductivities are shown in Figs. 5(a) and 5(b) for the solution and film 


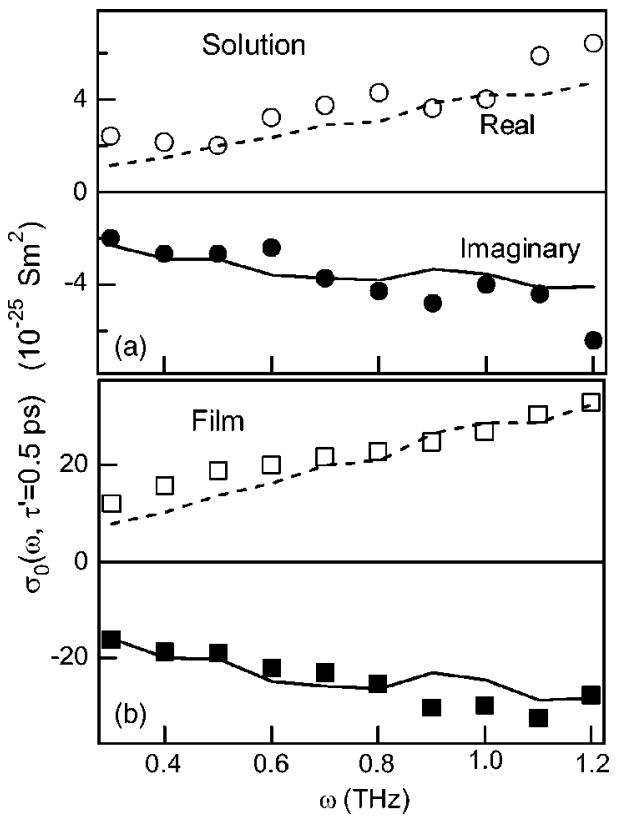

FIG. 5. (a) The frequency-dependent complex conductivity at the sample interface, normalized to initial surface excitation density (see text) measured in solution $0.5 \mathrm{ps}$ after excitation. The measured conductivity has the characteristics associated with dispersive transport of free charges: significant real and imaginary parts, both increasing with frequency. The lines show the expected conductivity for a free charge, simulated following the formalism in Ref. 44. The best fit comes from a quantum efficiency $\phi \sim 0.5 \times 10^{-6}$, indicating that only a very small fraction of excitons dissociates into free charges on such short timescales. (b) The lines show the same simulation with $\phi \sim 3 \times 10^{-6}$, indicating that the close proximity of neighboring chains in the film sample facilitates exciton dissociation.

samples, respectively. The short-time conductivity has the same basic characteristics in both samples: significant contributions of real and imaginary parts to the conductivity, both increasing with frequency. This type of signal has previously been identified as that principally from free charges, ${ }^{5}$ with significant real conductivity indicating charge carriers with a velocity in phase with the $\mathrm{THz}$ field (or, equivalently, the dissipation of energy from the $\mathrm{THz}$ field into the sample). The observed frequency dependence of the conductivity is characteristic of dispersive free-charge transport in a disordered medium, where localization caused by the disorder in the material structure causes non-Drude behavior. ${ }^{5}$ Indeed, the frequency dependence of the complex conductivity could be reproduced ${ }^{5}$ using a model of disorder-limited hole transport along the polymer chains, by the formalism introduced in Ref. 44 [plotted as lines in Figs. 5(a) and 5(b)]. The free-charge signal in solution is smaller than in the film by almost an order of magnitude (see Fig. 5). This implies that the quantum efficiency $(\phi)$ for charge carrier generation is smaller in solution. We can quantify this from the comparison between the data and the simulation, ${ }^{45}$ varying only $\phi$ to fit our data. Thus, we find $\phi=0.5 \pm 0.2 \times 10^{-6}$ and $\phi=3 \pm 1 \times 10^{-6}$ for the solution and thin-film samples, respectively.

The error stated in these values originates mainly from uncertainty in the laser fluence of the excitation pulse, which affects the absolute magnitude of the fitted charge generation efficiency. It should be noted, however, that since exactly the same fluence is used in both measurements, the ratio of experimentally determined quantum efficiencies in thin-film and solution samples can be determined with much larger accuracy and is found to be $\phi_{\text {film }} / \phi_{\text {sol }}=6 \pm 1$, assuming identical mobility of charges in both samples. Further, the extracted factor of 6 difference between the film and solution samples from comparison to the mobility model represents a lower limit: the model we use to calculate the frequencydependent mobility neglects both nontorsional defects (such as chemical defects resulting in a disruption of the $\pi$ conjugation $^{46}$ ) and charge coupling to the lattice (polaron formation) and, as such, overestimates the conductivity. These effects have been shown to be much larger in solid films than in solution: From comparison of the model to measured mobilities at $\mathrm{GHz}$ frequencies, it was demonstrated that the model overestimates conductivity by a factor of 10-100 in solution ${ }^{4}$ and an additional order of magnitude in film samples. ${ }^{47}$ Correcting for this, it is evident that free charges are more efficiently generated in the thin-film sample $\left(\phi \sim 10^{-3}\right)$ than in solution $\left(\phi \sim 10^{-5}\right)$. It should be noted that, compared to $\mathrm{GHz}$ frequencies, we are much less sensitive to defects at $\mathrm{THz}$ frequencies as more local transport is probed, so that these numbers (and the ratio of film to solution generation efficiencies) constitute upper limits. ${ }^{48}$

The relatively large value for $\phi$ in the film sample (2 orders of magnitude higher than in the solution sample) suggests that the close proximity of neighboring chains in the film sample facilitates hot exciton dissociation by allowing the resulting electron and hole charges to escape on separate chains and minimizing any subsequent interaction. This mechanism agrees well with recent work of Lipson et al. ${ }^{11}$ who observe strong morphological effects on the photocurrent in PPV film samples. The interchain escape mechanism is not possible in solution due to the large nearest-neighbor distances $(\sim 200 \AA)$, so that the separated charges must reside on the same chain. This reasoning also explains why significant real conductivity is observed on longer timescales $\left(\tau^{\prime}>2 \mathrm{ps}\right)$ in the films sample [see both Figs. 6(b) and 7(b)], indicating that at least some free charges have not recombined on these longer timescales, while in the solution sample the real component of the conductivity effectively decays to zero within 1-2 ps. It should be noted that even the small number of free charges observed on subpicosecond time scales in the solution sample may also result from chain-chain interactions: a small amount of aggregation may lead to interchain dissociation (though, for a good solvent such as benzene, this is at most a weak effect $\left.{ }^{26}\right)$. Thus, a perfectly isolated polymer chain may not show any freecharge generation, even on subpicosecond timescales. Reversely, in a perfectly organized film sample the free-charge yield might be much larger and close to the $100 \%$ generation efficiency, as, e.g., observed by Hegmann et al. for organic single crystals. ${ }^{49}$

The observed linear fluence dependence on both solution and film samples indicates that the probability per excitation of electron-hole dissociation is constant. This suggests that the probability of exciting a hot exciton in close proximity to a dissociation site is comparable to any other site on the 


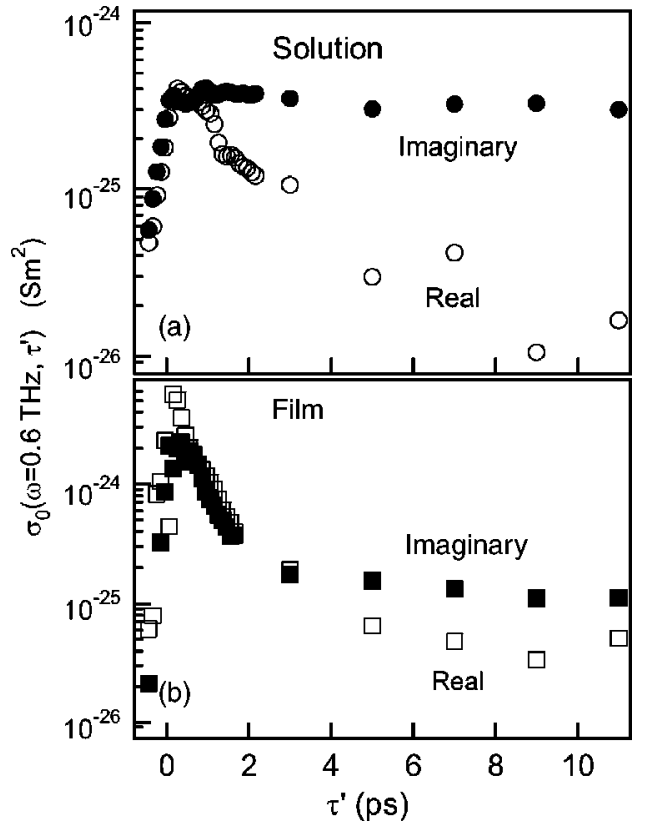

FIG. 6. (a) Magnitudes of the real and imaginary parts of the conductivity as a function of time $\tau^{\prime}$ after photoexcitation, measured at $0.6 \mathrm{THz}$ (the central frequency of the THz pulse), in the solution sample. The signal rises in around $300 \mathrm{fs}$, and in the first picosecond after photoexcitation both real and imaginary parts, corresponding to signal from free charges and bound excitons respectively, are significant. The real conductivity decays to effectively zero within $\sim 5 \mathrm{ps}$, while the imaginary component persists for around a nanosecond. (b) The same measurement on the film sample. Here, the initial conductivity is much higher, indicating a higher concentration of free charges. These persist on longer timescales, giving significant real conductivity after $5 \mathrm{ps}$. The imaginary component of conductivity decays within $150 \mathrm{ps}$, in agreement with the exciton lifetime in MEH-PPV films (Ref. 51).

polymer chain and that the dissociation probability decreases quickly with time after excitation before diffusion can play a role in the process. This picture fits in well with recently established hot exciton dissociation mechanisms, ${ }^{24,25}$ where the excess excitation energy is available for dissociation within $\sim 100$ fs after excitation. We also observe a weak dependence in the dissociation probability on excitation energy dependence. ${ }^{5}$ Such a non-Boltzmann dependence possibly indicates that the location of the excitation event is more important, in terms of exciton dissociation probability, than the extra energy given to the system. This idea is corroborated by the effect we see when we dope the film sample with PCBM, ${ }^{5}$ an electron scavenging molecule. This effectively increases the number of dissociation sites, and we observe almost an order-of-magnitude increase in free-charge generation, even on subpicosecond timescales.

While the chain-chain interactions seem to be essential to the mechanism of generation and survival of mobile charges, the similar frequency dependence (which is a probe of the length scales involved in disorder-dominated charge transport) in both samples suggests we are observing intrachain transport. Nevertheless, we cannot rule out the interchain transport on long timescales. In fact, since the polymer chains are not infinite in length, interchain transport

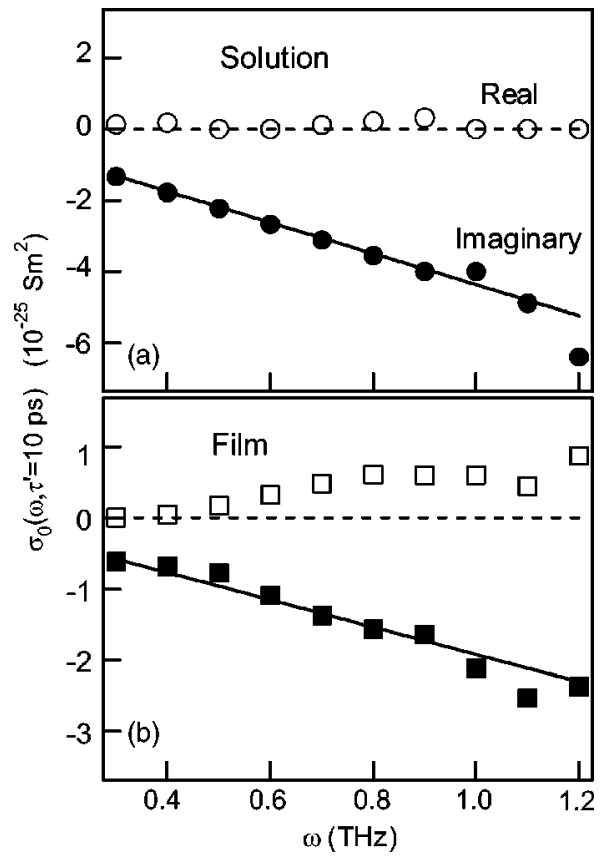

FIG. 7. The normalized frequency-dependent complex conductivity measured in solution (a) and film (b) samples, $10 \mathrm{ps}$ after excitation. At these times, the conductivity is predominantly imaginary: the solid and dashed lines show the response expected for excitons with polarizability $\alpha_{\text {sol }} \sim 1950 \pm 640 \AA^{3}$ and $\alpha_{\text {film }} \sim 770 \pm 180 \AA^{3}$, respectively; i.e., the average polarizability of excited species at these times is found to be 3 times larger in solution than in the film.

is required for there to be any dc conductivity (observed in such polymer films ${ }^{50}$ ). However, the $\mathrm{THz}$ field probes the high-frequency mobility on relatively shallow potential energy surfaces; clearly, transport along the polymer carbon backchain is highly favored over intrachain transport and the $\mathrm{THz}$ field of $1 \mathrm{kV} / \mathrm{cm}$ is too small to pull charges over an energy barrier. In other words, interchain hopping rates lie well below the $\mathrm{THz}$ range and outside our frequency window.

\section{B. Long-time conductivity: Exciton states}

The real part of the photoconductivity in both solution and film samples decays over 1-2 orders of magnitude within $\tau^{\prime}=2 \mathrm{ps}$, as shown in Fig. 6. The remaining signal is predominantly imaginary-see Fig. 7-and is dominated by bound (exciton like) species ${ }^{5}$ : the $\mathrm{THz}$ field can only induce an elastic deformation of the electron-hole wave functions (or, equivalently, a perturbation of the electron-hole pair separation) and does not give rise to any energy dissipation. The resulting imaginary conductivity decays more slowly ( $\sim 150 \mathrm{ps}$ and $\sim 1 \mathrm{~ns}$ for the film and solution, respectively, which agrees well with exciton lifetimes in these materials from transient luminescence ${ }^{51,52}$ ). As we are probing at frequencies $(1 \mathrm{THz}=4.1 \mathrm{meV})$ well below any excitonic resonances $^{53}$ and at times short compared to decay of the signal ( $10 \mathrm{ps}$ compared to $\sim 150 \mathrm{ps}$ and $\sim 1 \mathrm{~ns}$ ), the signal is determined by the average dc polarizability $\left(\alpha_{a v}\right)$ of bound 
species in the material and the instantaneous excitation density $\left(N_{0}\right)$. Indeed, in Fig. 7 we can model the response using the Clausius-Mossotti equation, which relates $\alpha_{a v}$ to the lowfrequency conductivity ${ }^{54}$ :

$$
\frac{n_{T H z}^{2}-1}{n_{T H z}^{2}+2}+\frac{\alpha_{a v} N_{0}}{3}=\frac{i \omega \varepsilon_{0}\left(n_{T H z}^{2}-1\right)-\sigma_{0}}{i \omega \varepsilon_{0}\left(n_{T H z}^{2}+2\right)-\sigma_{0}},
$$

where the low-frequency refractive indices $\left(n_{T H z}\right)$ are again and 1.51 and 1.78 for MEH-PPV films ${ }^{29}$ and benzene, respectively. Note that $\alpha^{a v}$ is simply proportional to the signal size of the imaginary conductivity at long times. Comparison with the data [see lines in Figs. 7(a) and 7(b)] yields $\alpha_{\text {sol }}^{a v} \sim 1950 \pm 640 \AA^{3}$ and $\alpha_{\text {film }}^{a v} \sim 770 \pm 180 \AA^{3}$, assuming a quantum efficiency of exciton formation of unity. These values are comparable to those found in previous measurements on PPV samples, in the range 800-3333 $\AA^{3}$ (see Ref. 55 for a summary), and similar to the theoretical estimate of $1000 \AA^{3}$ for an exciton on an isolated PPV chain. ${ }^{56}$ The errors in the extracted polarizabilities again originate mainly from uncertainty in the measured excitation fluence, which is identical in both measurements. Hence, the ratio of the solution and film polarizabilities, $\alpha_{\text {sol }}^{a v} / \alpha_{\text {film }}^{a v}=2.5 \pm 0.3$, can be determined with good accuracy. In solution, $\alpha_{\text {sol }}^{a v}$ $\sim 1950 \pm 640 \AA^{3}$ implies that the excitons are, on average, fairly weakly bound. For an exciton with a polarizability of this order [binding energy $\sim 0.8 \mathrm{eV}$ (Ref. 56)] the wave function is spread over several monomer units [root-meansquare distance between electron and hole $\sim 11 \AA$ (Ref. 56)], in agreement with observations on other $\pi$-conjugated systems. ${ }^{57,58}$ In the film, the substantially smaller polarizability suggests that the particles are on average more localized $(<11 \AA)$. The most obvious difference between the samples is the interchain distance: several authors have suggested that interchain species exist in significant numbers in film samples of PPV polymers, ${ }^{7,8}$ though this is certainly controversial. ${ }^{9}$ Our results indicate that any interactions with the neighboring chains do not, as one might expect, significantly increase delocalization. On the contrary, a decrease in polarizability in the film is observed, which can be explained by the increased disorder of the polymer chains in the film: the increased restriction placed on the polymer chains leads to a shorter average effective conjugation length, which in turn limits the exciton localization. ${ }^{14,57}$

These results show that the differences in morphology have a pronounced effect on the ultrafast photophysics of polymer semiconductors. This is in agreement with recent work by several other groups. ${ }^{13,28,59-62}$ These findings have implications for the design and application of polymers in electro-optic devices ${ }^{1}$ : these devices work by converting light to excitons and/or charges and vice versa. Hence, by controlling interchain distances, properties that are important to the operation of such devices can be modified. In particular, it is clear that chain morphology plays a key role in the ultrafast exciton dissociation into separated free charges. Morphological effects have also been observed in polymer solar cells, where thermally induced changes can result in a significant increase in device efficiency. ${ }^{63-65}$ Other devices, such as polymer light-emitting diodes, rely on the conversion of free electrons and holes into light emission. For these devices, the presence of weakly emissive interchain species greatly limits the efficiency of this process. ${ }^{6,28,66}$ Hence, for efficient luminescent devices, ${ }^{67}$ interchain effects are usually undesirable.

\section{SUMMARY}

To conclude, we have measured the $\mathrm{THz}$ frequencydependent conductivity on subpicosecond timescales of a solution and thin-film sample of a model semiconducting polymer (MEH-PPV). This allows us to study interchain effects on the ultrafast processes that follow photoexcitation. Our results indicate that the close proximity of nearest-neighbor chains in the film sample facilitates exciton dissociation on subpicosecond timescales by allowing the resulting electron and hole charges to escape on separate chains and permitting significant real conductivity even on much longer timescales. In solution, significantly fewer charges are photogenerated, but the increased freedom of the polymer chains results in a larger conjugation length and an exciton with greater spatial extent and corresponding larger average polarizability than in the film sample.

\section{ACKNOWLEDGMENTS}

The authors thank F. Wang and T. F. Heinz for helpful discussions and R. C. V. van Schie and P. Schakel for excellent technical support. This work is part of the research program of the "Stichting voor Fundamenteel Onderzoek der Materie" (FOM), which is financially supported by the "Nederlandse organisatie voor Wetenschappelijk Onderzoek" (NWO).
*FAX: +31-71-5274451.

Electronic address: e.hendry@chem.leidenuniv.nl

${ }^{1}$ M. Angelopoulos, IBM J. Res. Dev. 45, 57 (2001).

${ }^{2}$ B. J. Schwartz, Annu. Rev. Phys. Chem. 54, 141 (2003).

${ }^{3}$ A. R. Inigo, H. C. Chiu, W. Fann, Y. S. Huang, U. S. Jeng, C. H. Hsu, K. Y. Peng, and S. A. Chen, Synth. Met. 139, 581 (2003).

${ }^{4}$ R. Hoofman, M. P. Haas, L. D. A. Siebbeles, and J. M. Warman, Nature (London) 392, 54 (1998).

${ }^{5}$ E. Hendry, J. M. Schins, L. P. Candeias, L. D. A. Siebbeles, and
M. Bonn, Phys. Rev. Lett. 92, 196601 (2004).

${ }^{6}$ L. J. Rothberg, A. Safonov, I. D. W. Samuel, and W. L. Barnes, Synth. Met. 80, 41 (1996).

${ }^{7}$ M. Yan, L. G. Rothberg, F. Papadimitrakopoulos, M. E. Galvin, and T. M. Miller, Phys. Rev. Lett. 72, 1104 (1994).

${ }^{8}$ J. G. Muller, U. Lemmer, and J. Feldmann, Phys. Rev. Lett. 88, 147401 (2002).

${ }^{9}$ B. Kraabel, V. I. Klimov, R. Kohlman, S. Xu, H. L. Wang, and D. W. McBranch, Phys. Rev. B 61, 8501 (2000). 
${ }^{10}$ A. Ruini, M. J. Caldas, G. Bussi, and E. Molinuri, Phys. Rev. Lett. 88, 206403 (2002).

${ }^{11}$ S. M. Lipson, J. N. Coleman, A. Drury, D. F. O’Brien, W. J. Blau, A. J. Cadby, P. A. Lane, and D. D. C. Bradley, J. Appl. Phys. 95, 6138 (2004).

${ }^{12}$ M. Wohlgenannt, X. M. Jiang, and Z. V. Vardeny, Phys. Rev. B 69, 241204(R) (2004).

${ }^{13}$ M. Ariu, M. Sims, M. D. Rahn, J. Hill, A. M. Fox, D. G. Lidzey, M. Oda, J. Cabanillas-Gonzalez, and D. D. C. Bradley, Phys. Rev. B 67, 195333 (2003).

${ }^{14}$ A. J. Cadby, P. A. Lane, H. Mellor, S. J. Martin, M. Grell, C. Giebeler, and D. D. C. Bradley, Phys. Rev. B 62, 15604 (2000).

${ }^{15}$ T. Q. Nguyen, V. Doan, and B. J. Schwartz, J. Chem. Phys. 110, 4068 (1999).

${ }^{16}$ T. Q. Nguyen, I. B. Martini, J. Liu, and B. J. Schwartz, J. Phys. Chem. B 104, 237 (2000).

${ }^{17}$ S. Xu, V. I. Klimov, B. Kraabel, H. Wang, and D. W. McBranch, Phys. Rev. B 64, 193201 (2001).

${ }^{18}$ T. Q. Nguyen, I. B. Martini, J. Liu, and B. J. Schwartz, J. Phys. Chem. B 104, 237 (2000).

${ }^{19}$ S. V. Frolov, Z. Bao, M. Wohlgenannt, and Z. V. Vardeny, Phys. Rev. Lett. 85, 2196 (2000).

${ }^{20}$ S. Stagira, M. Nisoli, G. Lanzani, and S. D. Silvestri, Phys. Rev. B 64, 205205 (2001).

${ }^{21}$ H. D. Burrows, M. d. G. Miguel, A. P. Monkman, I. Hamblett, and S. Navaratnam, J. Mol. Struct. 563, 41 (2001).

${ }^{22}$ B. Kraabel, D. McBranch, N. S. Sariciftci, and A. J. Heeger, Phys. Rev. B 50, 18543 (1994).

${ }^{23}$ C. Silva, A. S. Dhoot, D. M. Russell, M. A. Stevens, A. C. Arias, J. D. MacKenzie, N. C. Greenham, R. H. Friend, S. Setayesh, and K. Müllen, Phys. Rev. B 64, 125211 (2001).

${ }^{24}$ V. I. Arkhipov, E. V. Emelianova, and H. Bässler, Phys. Rev. Lett. 82, 1321 (1999).

${ }^{25}$ D. M. Basco and E. M. Conwell, Phys. Rev. B 66, 155210 (2002).

${ }^{26}$ G. H. Gelinck, E. G. J. Staring, D. H. Hwang, G. C. W. Spencer, A. B. Holmes, and J. M. Warman, Synth. Met. 84, 595 (1997).

${ }^{27}$ H. A. Mizes and E. M. Conwell, Phys. Rev. B 50, R11 243 (1994).

${ }^{28}$ S. Vaidyanathan, H. Dong, and M. E. Galvin, Synth. Met. 142, 1 (2004).

${ }^{29}$ J. E. Mark, Polymer Data Handbook (Oxford University Press, New York, 1999), p. 722.

${ }^{30}$ M. C. Beard, G. M. Turner, and C. A. Schmuttenmaer, J. Phys. Chem. B 106, 7146 (2002).

${ }^{31}$ M. C. Beard, G. M. Turner, and C. A. Schmuttenmaer, Phys. Rev. B 62, 15764 (2000).

${ }^{32}$ G. Gallot and D. Grischkowsky, J. Opt. Soc. Am. B 16, 1204 (1999).

${ }^{33}$ J. T. Kindt and C. A. Schmuttenmaer, J. Chem. Phys. 110, 8589 (1999).

${ }^{34}$ J. Shan, F. Wang, E. Knoesel, M. Bonn, and T. F. Heinz, Phys. Rev. Lett. 90, 247401 (2003).

${ }^{35}$ E. Knoesel, M. Bonn, J. Shan, F. Wang, and T. F. Heinz, J. Chem. Phys. 121, 394 (2004).

${ }^{36}$ N. V. Smith, Phys. Rev. B 64, 155106 (2001).

${ }^{37}$ H. K. Nienhuys and V. Sundström (unpublished).

${ }^{38}$ M. C. Beard and C. A. Schmuttenmaer, J. Chem. Phys. 114, 2903 (2001).

${ }^{39}$ M. C. Beard, G. M. Turner, and C. A. Schmuttenmaer, J. Appl.
Phys. 90, 5915 (2001).

${ }^{40}$ J. M. Schins, E. Hendry, M. Koeberg, H. G. Muller, and M. Bonn (unpublished).

${ }^{41}$ G. Gallot, J. Zhang, R. W. McGowan, T. I. Jeon, and D. Grischkowsky, Appl. Phys. Lett. 74, 3450 (1999).

${ }^{42}$ H. J. Bakker, G. C. Cho, H. Kurz, Q. Wu, and X. C. Zang, J. Opt. Soc. Am. B 15, 1795 (1998).

${ }^{43}$ M. Samoc, A. Samoc, B. Luther-Davies, Z. N. Bao, and P. L. Yu, J. Opt. Soc. Am. B 15, 817 (1998).

${ }^{44}$ F. C. Grozema, P. T. v. Duijnen, Y. A. Berlin, M. A. Ratner, and L. D. A. Siebbeles, J. Phys. Chem. B 106, 7791 (2002).

${ }^{45}$ The model in Ref. 44 simulates the mobility $(\mu)$ of a charge along the polymer backbone. This is related to the measured conductivity $\left(\sigma_{0}\right)$ and the quantum efficiency $(\phi)$ through $\sigma_{0}=\left(\phi N_{0}\right) \mu e$. Then, knowing the absorbed experimental fluence, which determines the excitation density at the sample surface $\left(N_{0}\right)$ through the absorption length of the light $\left(z_{0}\right)$ used in the analysis, we can completely fit our data by varying only $\phi$.

${ }^{46}$ H. Becker, H. Spreitzer, K. Ibrom, and W. Kreuder, Macromolecules 32, 4925 (1999).

${ }^{47}$ J. M. Warman, G. H. Gelinck, and M. P. d. Haas, J. Phys.: Condens. Matter 14, 9935 (2002).

${ }^{48}$ The GHz mobilities were obtained from pulsed radiolysis experiments (such as reported in Ref. 47). The carrier densities in these experiments were calculated assuming that all the energy deposited in the sample was used to generate charges. As this efficiency is generally much smaller than $100 \%$, this sets a conservative lower limit for the carrier mobilities and an upper limit for the quantum efficiencies estimated here.

${ }^{49}$ F. A. Hegmann, R. R. Tykwinski, K. P. H. Lui, J. E. Bullock, and J. E. Anthony, Phys. Rev. Lett. 89, 227403 (2002).

${ }^{50}$ C. H. Lee, G. Yu, and A. J. Heeger, Phys. Rev. B 47, 15543 (1993).

${ }^{51}$ G. R. Hayes, I. D. W. Samual, and R. T. Phillips, Phys. Rev. B 52, R11 569 (1995).

${ }^{52}$ G. H. Gelinck, Ph.D. thesis, Delft University of Technology Delft, The Netherlands, 1998, p. 81.

${ }^{53}$ A. Kohler, D. A. d. Santos, D. Beljonne, Z. Shuai, J. L. Bredas, A. B. Holmes, A. Kraus, K. Mullen, and R. H. Friend, Nature (London) 392, 903 (1998).

${ }^{54}$ N. W. Ashcroft and N. D. Mermin, in Solid State Physics (Saunders College, Philadelphia, 1976), p. 542.

${ }^{55}$ G. H. Gelinck, J. J. Piet, B. R. Wegewijs, K. Müllen, J. Wildeman, G. Hadziioannou, and J. M. Warman, Phys. Rev. B 62, 1489 (2000).

${ }^{56}$ J. W. v. d. Horst, P. A. Bobbert, P. H. L. D. Jong, M. A. J. Michels, L. D. A. Siebbeles, J. M. Warman, G. Gelinck, and G. Brocks, Chem. Phys. Lett. 334, 303 (2001).

${ }^{57}$ M. Knupfer, J. Fink, E. Zojer, G. Leising, and D. Fichou, Chem. Phys. Lett. 318, 585 (2000).

${ }^{58}$ G. Hadziioannou and P. F. v. Hutten, in Semiconducting Polymers (Wiley-VCH, Weinheim, 2000).

${ }^{59}$ T. G. Bjorklund, S. H. Lim, and C. J. Bardeen, Synth. Met. 142, 195 (2004)

${ }^{60}$ M. Fakis, G. Tsigaridas, I. Polyzos, V. Giannetas, and P. Persephonis, Phys. Rev. B 68, 035203 (2003).

${ }^{61}$ B. J. Schwartz, Annu. Rev. Phys. Chem. 54, 141 (2003).

${ }^{62}$ C. H. Tan, A. R. Inigo, W. Fann, P. K. Wei, G. Y. Perng, and S. A. Chen, Microb. Pathog 3, 81 (2002). 
${ }^{63}$ J. K. J. van Duren, X. N. Yang, J. Loos, C. W. T. Bulle-Lieuwma, A. B. Sieval, J. C. Hummelen, and R. A. J. Janssen, Adv. Funct. Mater. 14, 425 (2004).

${ }^{64}$ M. Drees, R. M. Davis, and J. R. Heflin, Phys. Rev. B 69, 165320 (2004)

${ }^{65}$ V. Dyakonov, Appl. Phys. A: Mater. Sci. Process. 79, 21 (2004).
${ }^{66}$ R. H. Friend, R. W. Gymer, A. B. Holmes, J. H. Burroughes, R. N. Marks, C. Taliani, D. D. C. Bradley, D. A. D. Santos, J. L. Brédas, M. Löglund, and W. R. Salaneck, Nature (London) 397 121 (1999).

${ }^{67}$ J. A. E. Wasey, A. Safonov, I. D. W. Samuel, and W. L. Barnes, Phys. Rev. B 64, 205201 (2001). 